\title{
Factors which Affect the Release of Newcastle Disease and Sendai Viruses from Infected Allantoic Cells
}

\author{
BY R. D. BARRY \\ University of Cambridge Department of Pathology, \\ Tennis Court Road, Cambridge
}

(Received 12 November 1964)

\begin{abstract}
SUMMARY
Some aspects of the adsorption and release of Newcastle disease and Sendai viruses were studied in excised pieces of allantois of uniform size. About $40 \%$ of any saturating dose of either virus was adsorbed. The $\%$ adsorption was not influenced by treatment with neuraminidase. Onestep growth curves indicated that the release process for both viruses was linear. The rate of release and the burst size were influenced by the composition of the medium used for maintaining the allantoic cells. Increases in the multiplicity of infection decreased the length of the latent period. Treatment of allantoic cells with suitable doses of actinomycin D or ultraviolet irradiation also affected the length of the latent period. These agents may increase the susceptibility of cells to infection by affecting DNAdirected mechanisms concerned in the control of nucleic acid synthesis.
\end{abstract}

\section{INTRODUCTION}

Most myxoviruses grow well in the allantoic cells of the developing chick embryo. However, detailed quantitative studies have been made only with influenza virus. It has gradually become apparent that the myxoviruses are not a homogeneous group of closely related viruses, but consist of two distinct varieties (Waterson, 1962), of which the influenza viruses constitute one type. Newcastle disease virus (NDV) and Sendai virus are representatives of the other type of myxovirus, and it is the purpose of this paper to examine some quantitative aspects of their multiplication in allantoic cells.

\section{METHODS}

Strains of virus. The following strains of virus were used : NDV California 11914, and Sendai (originally obtained from The National Institute for Medical Research, Mill Hill, through the kindness of Dr A. Isaacs). Each strain consisted of an allantoic fluid material prepared and stored as previously described for influenza virus stocks (Barry, 1961).

Neuraminidase. Preparations of this enzyme were obtained from Behringwerke, Marburg, Germany. Titrated by the method of Burnet \& Stone (1947), they contained $10^{4}$ units of activity $/ \mathrm{ml}$.

Haemagglutination titrations. Haemagglutination (HA) titrations were done by the method of Fazekas de St Groth \& Graham (1955), except that $\mathbf{0 . 2 5} \mathrm{ml}$. volumes of a $0.5 \%(\mathrm{v} / \mathrm{v})$ fowl red cell suspension were used. When HA titrations were made in the presence of neuraminidase, trays were chilled to $2^{\circ}$ before the addition of cells. 
Media and solutions. PBS: phosphate-buffered saline (Dulbecco \& Vogt, 1954). SM: 'Standard Medium', a balanced salt solution (Fazekas de St Groth \& White, 1958) used for the maintenance of allantoic cells. 199: Parker's chemically defined medium 199 (Morgan, Morton \& Parker, 1950) was obtained from Burroughs Wellcome and Company, London.

Infectivity titrations. Infectivity was titrated by the method of Fazekas de St Groth \& White (1958), except that medium 199 was substituted for SM in the maintenance of membrane pieces.

The experimental system. Experiments were made with pieces of allantois-onshell, $1 \mathrm{~cm} .^{2}$ in size, obtained from 11-day or 12-day chick embryos. Barry (1961) described in detail the preparation and handling of such pieces. The pieces were placed individually in cups of large Perspex trays. Each cup contained $0.7 \mathrm{ml}$. of the appropriate medium, and virus was added in $0.02 \mathrm{ml}$. drops. Trays were then incubated on a shaking machine in a constant temperature room at $35^{\circ}$. Measurements of the uptake of virus, or of the yield of virus were determined by HA titration. Except where otherwise stated, each experimental point represents the average value obtained from four replicate pieces. For any particular experimental conditions, the yields of individual pieces showed little variation, e.g. 24 pieces, incubated in SM and infected with a saturating dose of NDV, produced an average yield $18 \mathrm{hr}$ later of $2^{5.4 \pm 02}$. The range of individual values was from $2^{5 \cdot 2}$ to $2^{5 \cdot ?}$.

Actinomycin. The actinomycin D was kindly provided by Dr J. Merry (Merck, Sharp and Dohme Ltd., Hoddesdon, Hertfordshire). Solutions containing $100 \mu \mathrm{g} . /$ ml. were prepared in distilled water and stored frozen. Suitable dilutions were prepared in SM or 199 media and then applied to cells.

Ultraviolet irradiation. Allantoic cell pieces washed several times in warm PBS were exposed for 10 sec., in open Petri dishes, to a 'chromatolite' portable ultraviolet lamp (Hanovia Ltd.), at a distance of $25 \mathrm{~cm}$. More than $95 \%$ of the output of this lamp had a wavelength $2537 \AA$.

\section{RESULTS}

\section{Estimation of multiplicity}

The adsorption of myxoviruses to host cells usually requires mucoproteins. In the case of influenza virus however, much of the virus attached to surface mucoproteins does not penetrate inside the host cells. To overcome this difficulty when measuring the multiplicity of infection, Cairns \& Edney (1952) treated cells, after exposure to virus, with neuraminidase, which releases all superficially bound virus. Under these conditions the actual amount of virus irreversibly bound by cells was always about $50 \%$ of the input. Before proceeding with studies of the growth cycle of NDV and Sendai, it was necessary to find how much virus was irreversibly bound to cells under different conditions of input. The $\%$ adsorption of these viruses to allantoic cells in the absence and presence of neuraminidase was, therefore, determined.

A dilution series of NDV allantoic fluid was prepared, and each dilution was added to batches of five replicate allantoic pieces. The membrane pieces were incubated for $90 \mathrm{~min}$. on the shaking machine, after which time the uptake of virus to cells was measured by determining the HA content of the supernatant medium 
(Fig. 1). Regardless of the actual amount of virus in the inoculum, about $40 \%$ of it became associated with the cells. In a similar experiment, identical results were obtained for Sendai virus.

To determine whether the \% adsorption of either virus was influenced by treatment with neuraminidase, batches of allantoic pieces were infected with various inputs of virus, and adsorption allowed to proceed for $90 \mathrm{~min}$. when each batch was

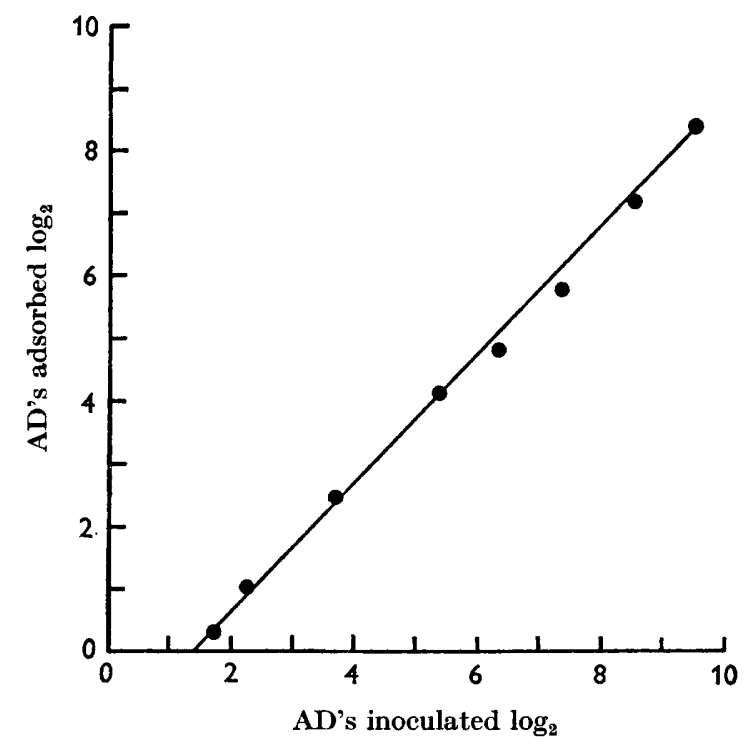

Fig. 1. The relation between the amount of virus inoculated and the amount adsorbed by pieces of allantois. One agglutinating dose (AD) of virus is equivalent to $10^{6 \cdot 3}$ ID50.

Table 1. The effect of neuraminidase on the percentage adsorption of Sendai virus to allantoic cells

\begin{tabular}{|c|c|c|c|c|c|}
\hline \multirow{2}{*}{$\begin{array}{c}\text { Input } \\
\text { no. }\end{array}$} & \multicolumn{3}{|c|}{$\begin{array}{l}\text { HA content/0.25 ml. } \\
\text { supernatant fluid }\left(\log _{2}\right)\end{array}$} & \multicolumn{2}{|c|}{$\%$ adsorption } \\
\hline & Controls & Treated* & Untreated & Treated* & Untreated \\
\hline 1 & $6 \cdot 3$ & $5 \cdot 4$ & $\mathbf{5 \cdot 5}$ & 46 & 40 \\
\hline 2 & $4 \cdot 7$ & $3 \cdot 9$ & 4.0 & 42 & 38 \\
\hline 3 & $3 \cdot 5$ & $2 \cdot 8$ & $2 \cdot 6$ & 38 & 46 \\
\hline 4 & $2 \cdot 2$ & 1.5 & 1.5 & 39 & 39 \\
\hline
\end{tabular}

* Ninety minutes after addition of virus, treated allantoic cell pieces were exposed to 100 units neuraminidase for a further 60 min.

divided into two. Half of the pieces were treated with 100 units neuraminidase for $1 \mathrm{hr}$ while the remainder were not. Controls consisted of $0.7 \mathrm{ml}$. volumes of medium containing virus but not allantoic pieces. At $2 \mathrm{hr}$ after infection, the uptake of virus was determined by HA titration. The amount of Sendai virus irreversibly bound to host cells was uninfluenced by neuraminidase, and was about $40 \%$ of the total inoculum (Table 1 ). NDV behaved si ilarly.

Each allantoic piece consists of about $3 \cdot 3 \times 10^{5}$ surface allantoic cells (Cairns \& 
Fazekas de St Groth, 1957). The stocks of NDV and Sendai were fully infectious; i.e. the ID50/HA ratio of each was $10^{6 \cdot 3}$. The virus content of any inoculum was corrected to infectious units (IU) by subtraction of $0 \cdot 15 \log _{10}$ from its total ID50 content. A further $0 \cdot 4 \log _{10}$ was subtracted from the total ID50 content to allow for that proportion of the input not adsorbed. It was thus possible to obtain an estimate for the maximum adsorbed multiplicity of infectious units per cell for any input of virus.

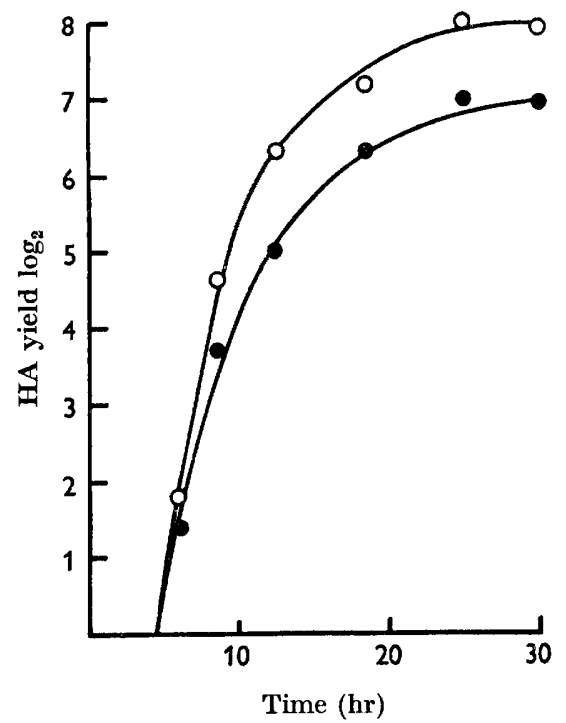

Fig. 2

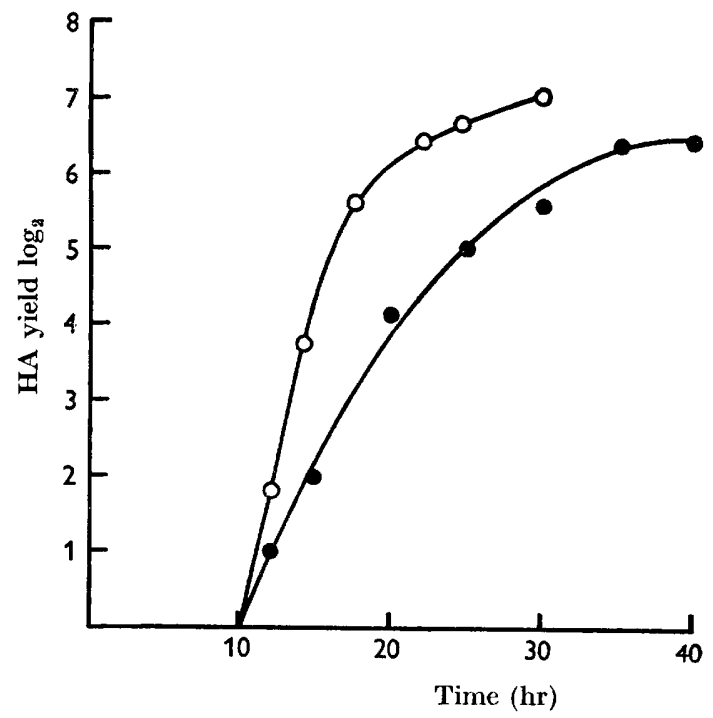

Fig. 3

Fig. 2. One-step growth curves for NDV in allantoic cells. Cells were infected with an estimated multiplicity of 4 IU per cell. $O=$ yields in $199 ; 0=$ yields in SM.

Fig. 3. One-step growth curves for Sendai virus in allantoic cells. Cells were infected with an estimated multiplicity of 4 IU per cell. $O=$ yields in $199 ; 0=$ yields in SM.

\section{One-step growth curves}

The growth of NDV and Sendai viruses was investigated by obtaining one-step growth curves. Experiments were performed by infecting allantoic pieces for $90 \mathrm{~min}$. with saturating doses of known virus content. After removal of the inoculum the pieces were washed in several changes of warm PBS. Fresh medium was then added to each piece and the trays replaced on the shaker. Subsequently, batches of infected pieces were removed at intervals and the medium in which they had been incubated was assayed for haemagglutinin. The cells received an input of virus estimated to provide an adsorbed multiplicity of $4 \mathrm{IU}$ per cell. The results indicated that for both viruses the maintenance medium of the host cells influenced release rate and burst size (Figs. 2, 3). Under optimal conditions, each membrane piece is capable of producing about $200 \mathrm{HA}$ units of NDV, and half this amount of Sendai. Assuming an ID50/HA ratio of about $10^{6 \cdot 3}$, each cell is then capable of producing approximately 1000 ID50 of NDV, or 500 ID50 of Sendai virus. 


\section{The effect of multiplicity of infection}

Although individual allantoic cells infected with influenza virus release the bulk of their yield over a short period of time, considerable variation exists between cells in the time of onset of this process (Cairns, 1957). This variation can be decreased by increasing the absorbed multiplicity of infection per cell. In a one-step growth experiment, reduction in asynchrony increases the rate of release. However, increasing the multiplicity of infection does not affect the time of onset of the

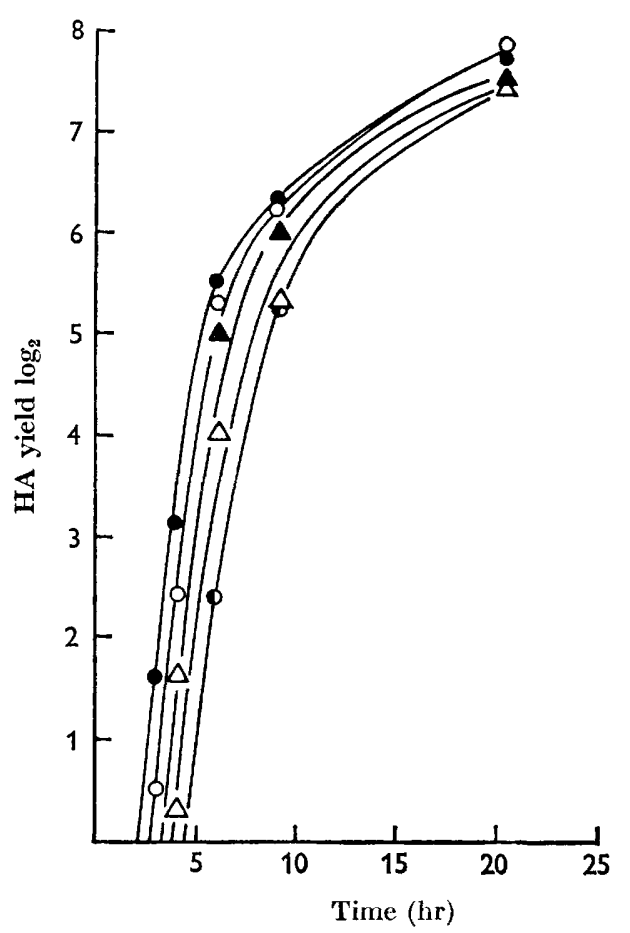

Fig. 4

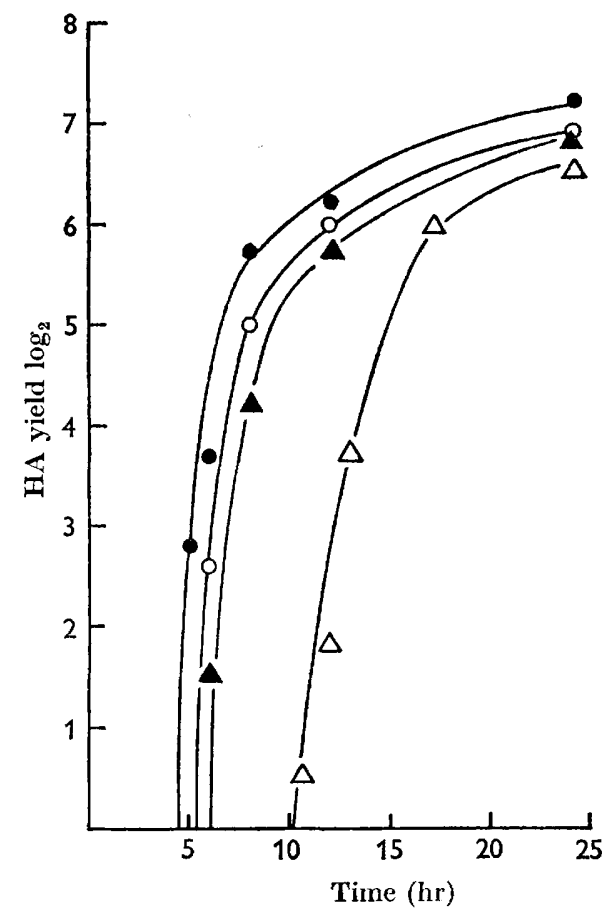

Fig. 5

Fig. 4. The effect of adsorbed multiplicity on the length of the latent period of NDV multiplication. From left to right, estimated adsorbed multiplicities of infection of approximately $500,120,30,8$ and 2 IU per cell, respectively.

Fig. 5. The effect of adsorbed multiplicity on the length of the latent period of Sendai virus multiplication. From left to right, estimated adsorbed multiplicities of infection of, approximately, 1000,500, 120 and 10 IU per cell, respectively.

release process. To determine whether the release process of NDV or Sendai is similar to that of influenza virus, one-step growth curves were made with different multiplicities of infection. Five batches of allantoic pieces were prepared and incubated in medium 199. Each batch received a particular dose of NDV such that, after adsorption, approximate multiplicities of 500, 120, 30, 8 and 2 IU per cell were achieved. The experiment was incubated at $35^{\circ}$ on the shaker, and at suitable intervals after infection the HA yield of groups of pieces from each batch was measured. As the multiplicity of infection increased, the time of appearance of 
newly formed virus shortened, but the rate of virus release was not affected (Fig. 4). Consequently, the response of cells to increasing doses of NDV differs from that found with influenza virus.

The latent period of Sendai virus multiplication was also influenced by the adsorbed multiplicity of infection. Batches of allantoic pieces were infected with estimated multiplicities of 1000,500,120 and $10 \mathrm{IU}$ of Sendai virus per cell, and growth curves for each input were obtained. Increased multiplicity of infection clearly decreased the latent period (Fig. 5).

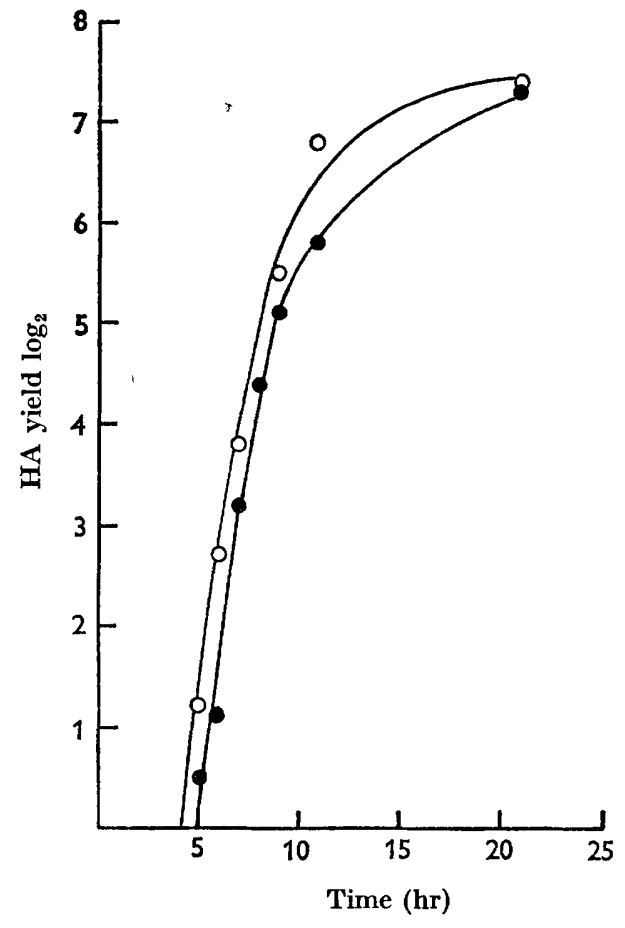

Fig. 6

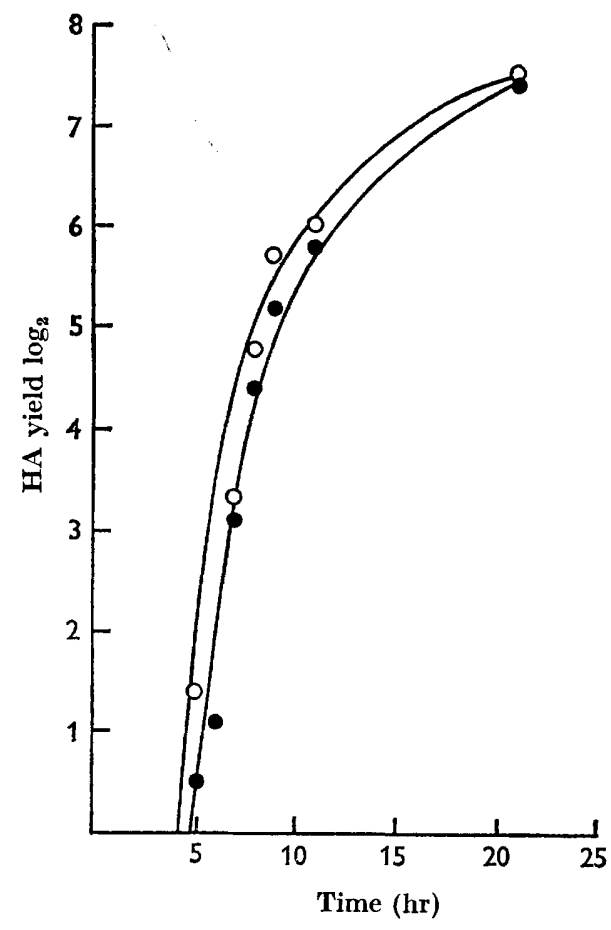

Fig. 7

Fig. 6. The effect of ultraviolet irradiation on the release of NDV. Allantoic cells were subjected to 10 sec. u.v. irradiation before infection with NDV at multiplicity of 2 IU per cell. $O=$ yields from irradiated cells; $O=$ yields from unirradiated control cells.

Fig. 7. The effects of actinomycin $\mathrm{D} 1 \mu \mathrm{g} . / \mathrm{ml}$. on the release of $\mathrm{NDV} . \quad O=$ yields from treated cells infected with 2 IU per cell; $0=$ yields from untreated cells infected with 2 IU per cell.

\section{The effects of inhibitors of nucleic acid synthesis}

The multiplication of influenza virus is suppressed by actinomycin $\mathrm{D}$ and by ultraviolet (u.v.) irradiation. These agents affect the function of cellular DNA (Barry, Ives \& Cruickshank, 1962; Barry, 1964). Doses of either inhibitor which decrease the yield of influenza by $99 \%$ do not affect the yield of either NDV or Sendai. In preliminary studies, it was found that treatment of allantoic cells with small doses of either inhibitor (1-2 $\mu \mathrm{g}$. $/ \mathrm{ml}$. actinomycin, up to 10 sec. of u.v. irradiation) apparently increased the yield of NDV. The nature of this effect was in- 
vestigated further by examining the effect of apparently stimulating doses of u.v. radiation or actinomycin D on the growth cycle of NDV and Sendai. One-step growth curves were obtained for both viruses in the presence and absence of stimulating doses of each agent. One batch of allantoic pieces was subjected to $10 \mathrm{sec}$. of u.v. irradiation before exposure to a dose of NDV giving an absorbed multiplicity of 2 IU/cell. The results were compared to those obtained from infected, unirradiated control pieces. This dose of u.v. radiation did not influence the size of the final yield or the release rate, but did influence the time of onset of virus release (Fig. 6).

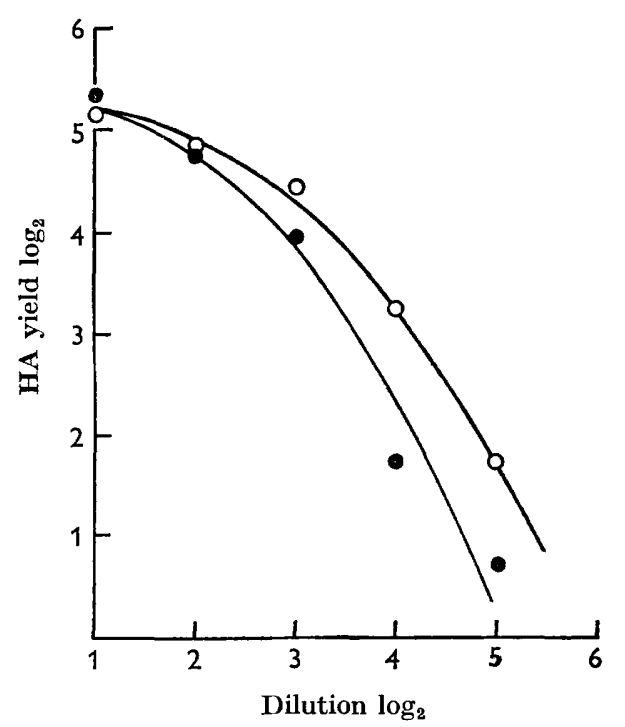

Fig. 8

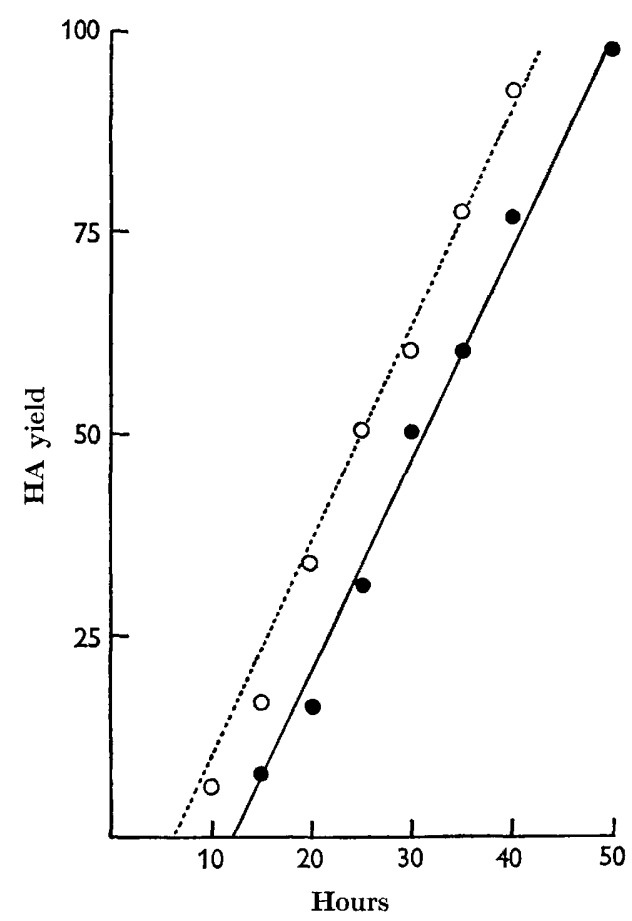

Fig. 9

Fig. 8. The effects of actinomycin D $1 \mu \mathrm{g} . / \mathrm{ml}$. on the amount of Sendai virus released from allantoic cells $10 \mathrm{hr}$ after infection with a range of input multiplicities. $O=$ yields from treated cells; $O=$ yields from untreated cells.

Fig. 9. The linearity of the release process of Sendai virus from allantoic cells when infected with $10 \mathrm{IU}$ per cell. $O=$ yields in the presence of actinomycin $\mathrm{D} 1 \mu \mathrm{g} . / \mathrm{ml}$.; $=$ yields in the absence of actinomycin $\mathrm{D}$.

Identical results were obtained with Sendai virus. Suitable concentrations of actinomycin also influenced the release rate. Cells were again infected at a multiplicity of $2 \mathrm{NDV}$ IU/cell. Half the pieces were incubated in actinomycin D (1 $\mu \mathrm{g} . / \mathrm{ml}$.) The results were the same as those obtained with $u . v$. radiation (Fig. 7 ), indicating that this substance at this concentration also shortened the latent period of infection. Similar results were obtained with Sendai virus. Much higher doses of either u.v. radiation or actinomycin $\mathbf{D}$ did not affect the total yield of NDV or Sendai, but the latent period is influenced only when small doses are used.

To demonstrate that inhibitor-induced shortening of the latent period occurred 
at a variety of input multiplicities, batches of allantoic pieces were infected with individual dilutions of Sendai virus, providing adsorbed multiplicities of 160,80 , 40, 20 and $10 \mathrm{IU}$ per cell. Each batch was divided in two, one half of the pieces receiving $1 \mu \mathrm{g} . / \mathrm{ml}$. actinomycin $\mathrm{D}$, the remaining pieces serving as controls. Infection was allowed to proceed for $10 \mathrm{hr}$ and then HA yields were determined. The release of newly formed virus occurred more rapidly from cells treated with actinomycin $\mathrm{D}$ than from the controls, although, as the multiplicity of infection increased, this effect was gradually obscured (Fig. 8).

\section{The susceptibility of host cells}

Suitable doses of actinomycin $\mathbf{D}$ or u.v. radiation make host cells respond to infection as though they had received a higher input of virus. In the examples shown in Figs. 6 and 7, the cells responded as though they received an input four times higher than that actually used. To determine whether this effect was attributable to an increase in the susceptibility of host cells to infection, each virus was titrated for infectivity in cells subjected to $10 \mathrm{sec}$. of u.v. irradiation, and the titres compared to those obtained in untreated cells. In those experiments, twofold dilutions of virus were prepared and $0.02 \mathrm{ml}$. volumes of each were inoculated into each of 50 allantoic pieces. Treatment of cells before infection with a stimulating dose of u.v. radiation resulted in an approximately twofold increase in the endpoints obtained for either virus (Table 2).

Table 2. The susceptibility of ultraviolet-irradiated allantoic pieces to infection

$\begin{array}{lcc}\text { Control } & \begin{array}{c}* \text { U.v. } \\ \text { irradiated }\end{array} \\ \text { Virus } & \text { Infectivity titre/0.25 ml. }\left(\log _{10}\right) \\ \text { NDV } & 8 \cdot 60 & 8.95 \\ \text { Sendai } & 8 \cdot 35 & 8 \cdot 63\end{array}$

* Pieces of allantois were exposed in open Petri dishes to irradiation at wavelength $2537 \AA$ for 10 sec. at a distance of $25 \mathrm{~cm}$.

\section{DISCUSSION}

The presence of adsorbed virus at the surface of host cells, and its subsequent elution, was an unexpected complication in some quantitative studies of influenza virus multiplication (Cairns, 1955), but could be avoided when the cells were pretreated with neuraminidase after adsorption (Cairns \& Edney, 1952). In the present study, it was found that, unlike influenza, the \% adsorption of any saturating dose of either NDV or Sendai virus was not complicated in this way. Only $40 \%$ of the total input was irreversibly bound by allantoic cells, whether or not neuraminidase was added. Consequently, estimates of multiplicity were based on the assumption that the whole $40 \%$ of irreversibly bound virus was adsorbed by cells, thus providing an upper limit to the likely number of adsorbed infectious units per cell.

One-step growth experiments provide a simple means of investigating the characteristics of the release process : burst size, rate of release, and time of onset of release may be determined. Each of these properties could be influenced experimentally. 
The rate of release and the size of the final yield depended on the nature of the medium used for the maintenance of the host cells. The best results were obtained with a complex medium containing purines, pyrimidines, amino acids and vitamins. Not only did release occur more rapidly in this medium than in a balanced salt solution, but with both viruses the final yield was about twice as high.

Individual cells infected with influenza virus apparently yield the bulk of their output over a short period of time; the onset of this release process can be synchronized by increasing the multiplicity of infection (Cairns, 1957). Consequently, the multiplicity of infection affects the release rate of influenza virus, without affecting its time of onset. The growth curves for NDV and Sendai viruses indicate a linear release process. This can be seen clearly in Fig. 9 and suggests that, once release begins, individual NDV and Sendai infected cells produce virus at a constant rate for many hours. Hence the release process for these viruses differs from that of influenza. Furthermore, increasing the multiplicity of infection does not affect the release rate of NDV or Sendai, although it does affect the time of onset of release. Consequently, NDV and Sendai resemble vesicular stomatitis virus (Cooper, 1958) and not inflenza virus, in that increasing the multiplicity results in shortening of the latent period.

Suitable doses of either actinomycin $D$ or u.v. radiation also affect the length of the latent period. The ability of actinomycin $\mathbf{D}$ to shorten the latent period of Sendai virus multiplying in human amniotic cells was reported (Bukrinskaya \& Zhdanov, 1963).

The possibility was considered that actinomycin $\mathbf{D}$ or u.v. radiation interfere with cellular nucleic acid synthesis, increasing the supply of precursors available for virus nucleic acid synthesis, so that virus development occurs more quickly in treated cells. The present experiments on the effects of different media were made to test this notion. However, variation in the supply of ingredients in the medium affected only the rate of release and not the time of onset. Attempts were also made to determine whether treatment with either actinomycin or u.v. radiation increased the intracellular nucleotide pool. The amount of trichloroacetic acid soluble nucleotides which could be extracted from both treated and control cells was determined spectrophotometrically (Newton, Dendy, Smith \& Wildy, 1962). No differences could be detected. Consequently, it was not possible to provide support for the idea that availability of substrates is an important factor in determining the time of onset of virus release.

The possibility was also considered that inhibitors of RNA synthesis might have affected the susceptibility of cells to infection. Suitable doses of either inhibitor changed the response of cells to a particular dose of virus in such a way that they responded as though they had received a dose which was four times higher. That this was at least in part due to a change in susceptibility was demonstrated in infectivity titrations using stimulating doses of u.v. radiation. The conventional infectious unit for both viruses used in this study represents about ten physical particles (Isaacs \& Donald, 1955). Under normal circumstances apparently only one particle in ten succeeds in infecting. Inhibitor treatment seemed to enable many otherwise undetected and non-functional virus particles to participate in multiplication. If this is so, not only may more particles be adsorbed than usually participate in the production of infection, but, given the opportunity, these particles may be capable 
of functioning normally. It seems reasonable to suppose that cells actively block the establishment of infection, and that on average only about one virus particle in ten succeeds. If cellular DNA function is impaired, the chance of any adsorbed particle initiating infection increases. Virus invasion may stimulate an active, DNAdirected response on the part of the cell to resist infection. The observation of Heller (1963) that actinomycin prevented the production of interferon by infected cells is in keeping with these findings and suggestions: interferon may represent the means by which cells attempt to control nucleic acid synthesis.

\section{REFERENCES}

BARRy, R. D. (1961). The multiplication of influenza virus. I. The formation of incomplete virus. Virology, 14, 389.

BARRY, R. D. (1964). The effects of actinomycin $D$ and ultraviolet irradiation on the production of fowl plague virus. Virology, 24, 563.

Barry, R. D., Ives, D. R. \& Cruickshank, J. G. (1962). Participation of deoxyribonucleic acid in the multiplication of influenza virus. Nature, Lond. 194, 1139.

Bukrinskaya, A. G. \& Zhdanov, V. M. (1963). Shortening by actinomycin D of the latent period of multiplication of Sendai virus. Nature, Lond. $200,920$.

Burnet, F. M. \& Stone, J. D. (1947). The receptor destroying enzyme of $V$. cholerae. Aust. J. exp. Biol. med. Sci. 25, 227.

CaIrns, H. J. F. (1955). Multiplicity reactivation of influenza virus. J. Immun. 75, 326.

CaIrNs, H. J. F. (1957). The asynchrony of infection by influenza virus. Virology, $3,1$.

CaIrns, H. J. F. \& EDney, N. (1952). Quantitative aspects of influenza virus multiplication. 1. The production of 'incomplete' virus. J. Immun. 69, 155.

Cairns, H. J. F. \& Fazekas de St Groth, S. (1957). The number of allantoic cells in the chick embryo. J. Immun. 78, 191.

CoOper, P. D. (1958). 'Shortened latency' as a result of multiple infection by vesicular stomatitis virus in chick cell culture. J. gen. Microbiol. 19, 340.

Dulbecco, R. \& Vogt, M. (1954). Plaque formation and isolation of pure lines with poliomyelitis virus. J. exp. Med. 99, 167.

Fazekas de St Groth, S. \& Graham, D. M. (1955). The production of incomplete virus particles among influenza strains. Chemical induction of the von Magnus phenomenon. Brit. J. exp. Path. 36, 205.

FazeKas De St Groth, S. \& White, D. O. (1958). An improved assay for the infectivity of influenza virus. J. Hyg., Camb. 56, 151.

Heller, E. (1963). Enhancement of chikungunya virus replication and inhibition of interferon production by actinomycin D. Virology, 21, 652.

IsaAcs, A. \& Donald, H. B. (1955). Particle counts of haemagglutinating viruses. $J$. gen. Microbiol. 12, 241.

Morgan, J. F., Morton, H. J. \& Parker, R. C. (1950). Nutrition of animal cells in tissue culture. I. Initial studies on a synthetic medium. Proc. Soc. exp. Biol. Med. 73, 1.

Newton, A., Dendy, P. P., Smith, C. L. \& Wildy, P. (1962). A pool size problem associated with the use of tritiated thymidine. Nature, Lond. 194, 886.

Waterson, A. P. (1962). Two kinds of myxovirus. Nature, Lond. 193, 1163. 